\title{
Research Paper \\ Effectiveness of a Mindfulness-Based Stress Reduction Program on Functional Disability of Patients with Multiple Sclerosis
}

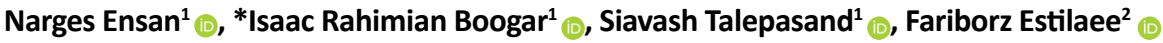

1. Deparment of Psychology, Faculty of Psychology \& Educational Sciences, Semnan University, Semnan, Iran. 2. Comprehensive Neurological Center of Dr. Estilaee, Neyshabur, Iran.

\begin{tabular}{|c|c|}
\hline $\begin{array}{l}\text { Use your device to scan } \\
\text { and read the article online }\end{array}$ & Citation: Ensan N, Rahimian Boogar I, Talepasand S, Estilaee F. [Effectiveness of a Mindfulness-Based Stress Reduction \\
\hline 口ifing & $\begin{array}{l}\text { Program on Functional Disability of Patients with Multiple Sclerosis (Persian)]. Journal of Arak University of Medical Sciences } \\
\text { (JAMS). 2019; 22(5):56-67. https://doi.org/10.32598/JAMS.22.5.56 }\end{array}$ \\
\hline Ifretsin & doi'https://doi.org/10.32598/JAMS.22.5.56 \\
\hline
\end{tabular}

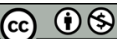

Article Info:

Received: 30 Jan 2019

Accepted: 28 Sep 2019

Available Online: 01 Dec 2019

Key words:

Mindfulness-based

stress reduction,

Functional disability,

Multiple sclerosis

\section{A B STRACT}

Background and Aim Multiple Sclerosis (MS) is an unpredictable disease that can change individuals' lifestyle. The purpose of this study was to investigate the effect of a Mindfulness-Based Stress Reduction (MBSR) program on functional disability of patients with MS.

Methods \& Materials The is an experimental study with pre-test/post-test/follow up design using control group. The study population consisted of all male and female patients with MS living in Neyshabur county, Iran. Of these, 47 were selected using a convenience sampling technique and randomly assigned into two groups of intervention $(n=22)$ and control $(n=25)$. The intervention group received MBSR therapy for 8 sessions, while the control group received no intervention. The follow-up was performed after one month. Subjects' functional disability was measured by using the World Health Organization Disability Assessment questionnaire. The collected data were analyzed using SPSS v.21 software usingrepeated measurements Analysis of Variance (ANOVA).

Ethical Considerations This study obtained its ethical approval from the Research Ethics Committee of Neyshabur University of Medical Sciences (code: IR. NUMS.REC.1397.004)

Results The results of univariate ANOVA showed the significant effect of time and the interaction effect of time and group on functional disability of samples. The results of between-group ANOVA a significant difference between the intervention and control groups in functional disability $(p<0.05)$. Pairwise comparison of the interaction effect of time and group showed a significant difference between the pre-test, post-test and follow-up scores $(P=0.001)$, but no significant difference was reported between the posttest and follow-up scores.

Conclusion The MBSR program could reduce the functional disability in patients with MS due to ease of treatment, faster patient education, and the short duration of intervention sessions..

\section{Extended Abstract}

\section{Introduction}

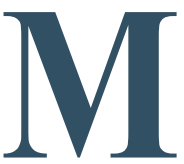

ultiple Sclerosis (MS) is a chronic, progressive inflammatory disease of the central nervous system that causes my-

$$
\text { ㄷ... }
$$

\section{* Corresponding Author:}

Isaac Rahimian Boogar, PhD.

Address: Deparment of Psychology, Faculty of Psychology \& Educational Sciences, Semnan University, Semnan, Iran.

Tel: +98 (23) 33624250

E-mail:i_rahimian@semnan.ac.ir 
ences in interacting with environmental factors and health conditions [10]. In recent years, scientists used non-pharmacological interventions in MS treatments. One of these methods is the mindfulness-based stress reduction (MBSR) program with a behavioral intervention based on self-focused attention [15]. It is an 8-week program focused on teaching a variety of mindfulness exercises including sitting meditation, body scan, walking meditation, loving meditation, and gentleness. In this method, the patients learn to increase self-compassion in a non-judgment way; they learn to increase moment-to-moment awareness rather than having automatic reaction as well as learning new ways of responding to situations [16]. In this study, the key question is whether MBSR therapy can affect functional disability in patients with MS.

\section{Materials and Methods}

The study population consisted of all male and female patients with MS in Neyshabur City, Iran $(\mathrm{N}=120)$. Of these, 47 were selected using a convenience sampling technique and randomly assigned into two groups of intervention $(n=22)$ and control $(n=25)$. Inclusion criteria were as follows: 1. Diagnosis of MS by the physician; 2. Age range of 20-55 years; 3. Reading and writing literacy; 4. Having MS over the past 6-12 months; 5 . Not receiving two psychological interventions at the same time; 6 . Having consent to participate in the study. Exclusion criteria were as follows: 1. History of psychiatric diseases and taking medications in the past year; 2. Having mental retardation, 3. Having suicidal thoughts and history of substance abuse and alcohol consumption; 4. Absence of more than two sessions in counseling sessions; 5. Having no cooperation during the interventions.

At first, the patients were matched for demographic characteristics (e.g. age, level of education, and the severity of disease) to control the confounding variables. The intervention group received MBSR program for 8 sessions, while the control group received no intervention. Follow-up was performed after one month. The subjects responded to the World Health Organization Disability Assessment questionnaire. It was developed by Aston (2010) to evaluate the functional disability in patients with chronic pains. This tool has 36 items assessing 6 subscales. The Cronbach's alpha coefficient for the whole questionnaire was 0.95 which indicates the instrument had good validity and reliability for assessment of disability. Repeated measures Analysis of Variance (ANOVA) was performed in SPSS V. 21 software for analyzing collected data.

\section{Results}

The results of univariate ANOVA showed the significant effect of time on functional disability $(\mathrm{F}=29.04, \mathrm{P}=0.001)$ as well as the significant effect of interaction effect of time and group on functional disability $(\mathrm{F}=26.18, \mathrm{P}=0.001)$. Furthermore, pretest scores were significantly different from posttest and follow-up scores $(\mathrm{P}=0.001)$; however, no significant difference was found between posttest and follow-

Table 1. Time and group effects of MBSR program on functional disability

\begin{tabular}{cccccccccc}
\hline Source & Test & Variable & $\begin{array}{c}\text { Sum of } \\
\text { Squares }\end{array}$ & Df & $\begin{array}{c}\text { Mean } \\
\text { Square }\end{array}$ & F & Sig. & $\begin{array}{c}\text { Eta } \\
\text { Squared }\end{array}$ & $\begin{array}{c}\text { Test } \\
\text { Power }\end{array}$ \\
\hline Time & $\begin{array}{c}\text { Greenhouse- } \\
\text { Geisser }\end{array}$ & $\begin{array}{c}\text { functional } \\
\text { disability }\end{array}$ & 81.4376 & 38.1 & 96.3166 & 04.29 & 001.0 & 39.0 & 00.1 \\
Time* group & $\begin{array}{c}\text { Greenhouse- } \\
\text { Geisser }\end{array}$ & $\begin{array}{c}\text { functional } \\
\text { disability }\end{array}$ & 32.3945 & 38.1 & 74.2854 & 18.26 & 001.0 & 36.0 & 00.1 \\
\hline
\end{tabular}
Journal of
Arak University of Medical Sciences

Table 2. ANOVA results (between-group effects)

\begin{tabular}{ccccccccc}
\hline Source & Variable & Sum of squares & Df & Mean square & F & Sig. & Eta squared & Test power \\
\hline Group & $\begin{array}{c}\text { functional } \\
\text { disability }\end{array}$ & 89.34745 & 1 & 89.34745 & & & & \\
Error & 38.9615 & 45 & 67.213 & 61.162 & 001.0 & 78.0 & 00.1 \\
\hline
\end{tabular}


up scores. This indicates the effectiveness of MBSR intervention on functional disability after intervention, which continued in the follow-up phase (Tables 1,2).

\section{Conclusion}

The purpose of this study was to investigate the effect of MBSR program on functional disability of MS. patients. The results showed that MBSR therapy can reduce the functional disability in patients with MS. Its effectiveness was continued in the follow-up phase.

Grossman et al. [26] believe that MBSR therapy can help people with MS to cope with life's challenges like functional impairment. By examining 150 patients with Secondary Progressive Multiple Sclerosis (SPMS), they showed that MBSR program for 8 weeks, compared to other conventional care methods, affects depression, fatigue, disability, and the Quality of Life and these effects are stable over a six-month follow-up. It can be argued that MBSR program leads to improved function of the patient with MS by reducing psychological stress indices and psychological distress. According to Mills and Allen [27], mindfulness to physical movements and functions in patients with MS serves as a coping strategy, and continuation of moment-to-moment awareness leads to improvement in patient's motion and function.

MBSR intervention is a self-help method for enhancing the physical and psychological functions in patients with MS. In this regard, Carlson [28] believe that this intervention reduces functional disability and causes multifaceted improvement in physical, social and cognitive functions of the patient by increasing the patient's awareness of the disease symptoms and conditions, and has a positive effect on the Quality of Life of the patient and his/her family and close relatives. The effectiveness of MBSR program reported in this study may be due to its ease of treatment, faster patient education, and the short duration of intervention sessions. It can also be concluded that these interventions can be used as a useful method to reduce the functional disability of MS patients.

\section{Ethical Considerations}

\section{Compliance with ethical guidelines}

This study obtained its ethical approval form the Research Ethics Committee of Neyshabur University of Medical Sciences (Code: IR.NUMS.REC.1397.4) and is a registered clinical trial (Code: IRCT20151228025732N33). All participants of the study signed a written consent form and were assured of the confidentiality of their information.

\section{Funding}

This study was extracted from a master thesis approved by Narges Ensan in Department of Clinical Psychology, Faculty of Psychology and Educational Sciences, Semnan University.

\section{Authors' contributions}

All authors met the writing standards of the International Committee of Medical Journal Publishers and all contributed equally to the writing of this article.

\section{Conflicts of interest}

The authors declare no conflict of interest.

\section{Acknowledgements}

The authors would like to thank the university, patients participated in the study and all those helped in conducting this study. 


\title{
اثربخشى مداخله كاهش استرس مبتنى بر ذهن آكاهى بر ناتوانى كاركردى بيمار ان مبتلابه مولتييل

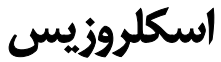

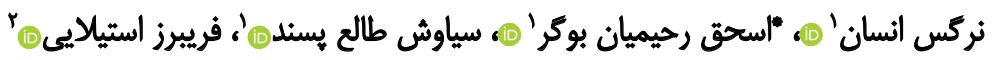 \\ I. كروه روانشناسى بالينى، دانشكده روانشناسى و علوم تربيتى، دانشعاه سمنان، سمنان، ايران. \\ r. مركز جامع مغز واعصاب دكثر استيلاتي، نيشابور، ايران.
}

\begin{abstract}
سيكين

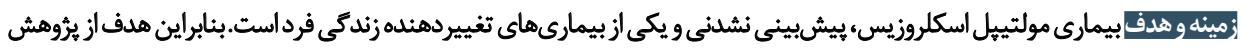

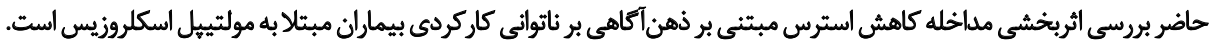

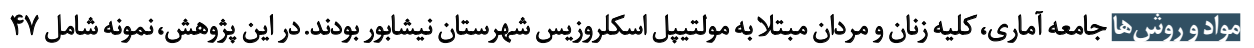

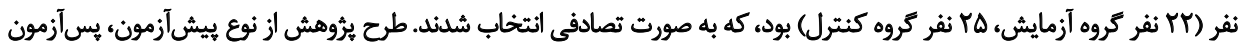

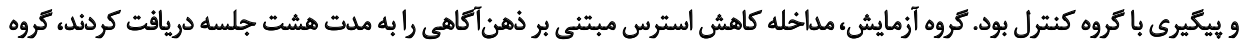

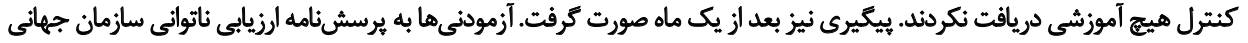

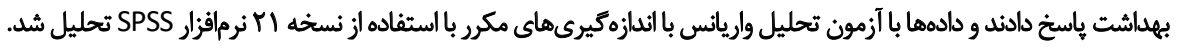

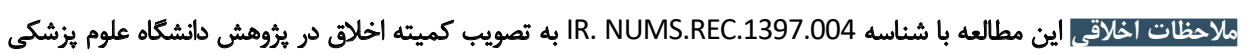

نيشابور رسيدهاست.

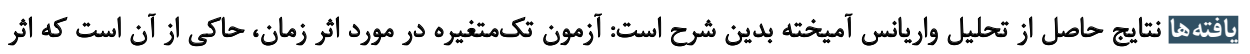

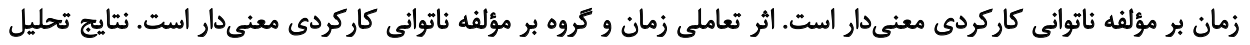

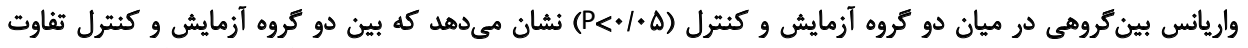

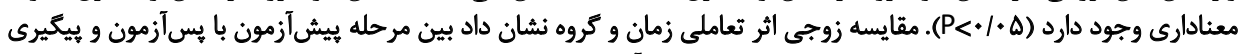

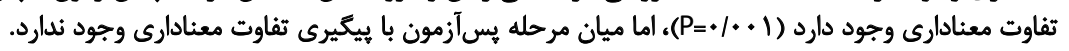

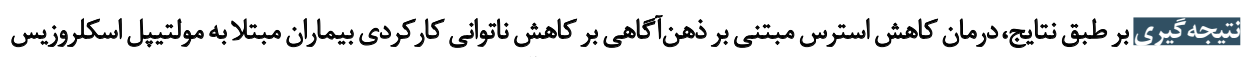

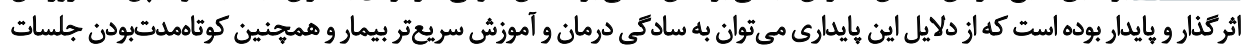

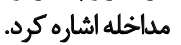

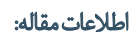

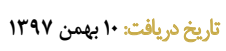
تاريخ بذيرشي: 9. مهر

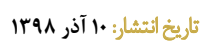

$: 00 \lg 15$

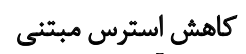
بر ذهن آكاهيى ، ناتوانى كاركردى ، مولتيبل اسكلروزيس

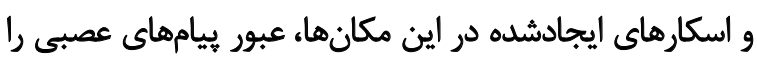
daves

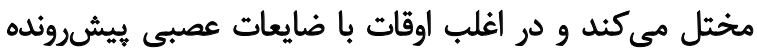

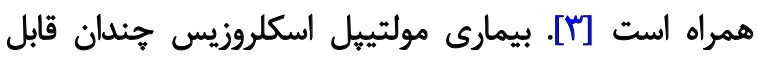

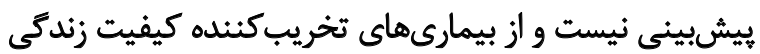

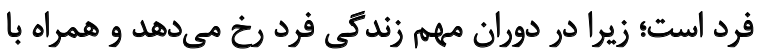

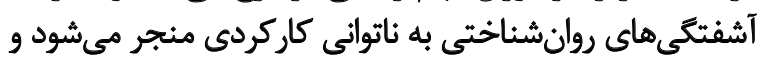

درمان قطعى نيز ندارد [†]

عوامل محيطى و رثنتيكى در استعداد ابتلا به مولتيخيل

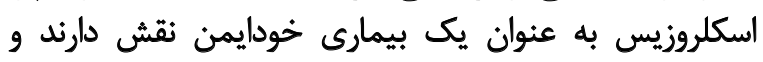

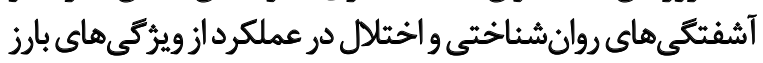
اين اختلال است [ه]. طبق آمار انجمن ملى مولتيبل اسكلروزيس

تغييير الكوى مركومير بيمارى، از بيمارىهاى حاد به سوى مناى

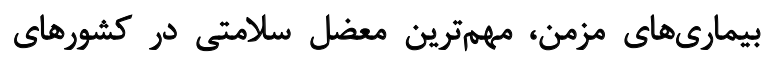

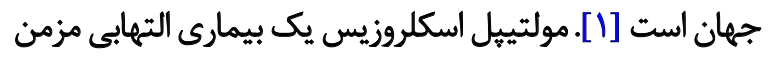

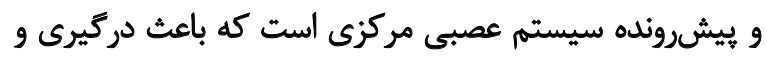

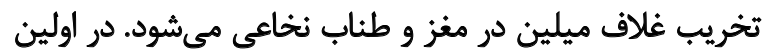

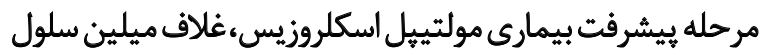

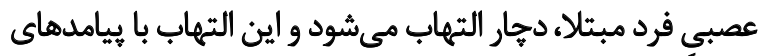

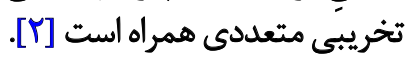
مولتييل اسكلروزيس با آسيبزدن به ميلين اعصاب مغز ونخاع 
برنامه كاهش استرس مبتنى بر ذهن آتاهي است. كاهش استرس

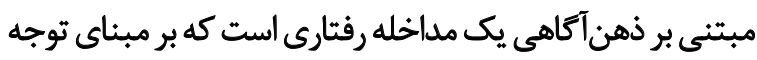

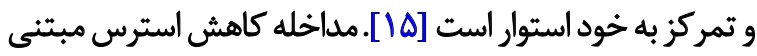

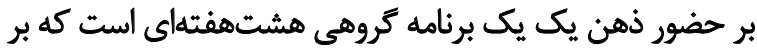

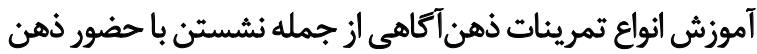

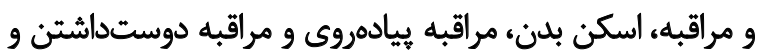

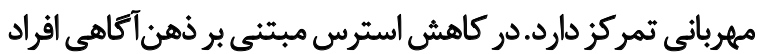

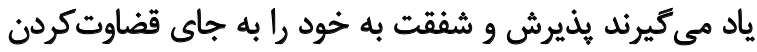

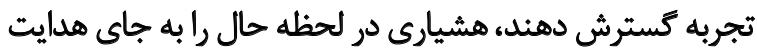

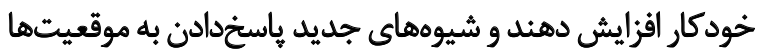
] افراكيرند [19].

افزايش ذهن آكاهى كه در حيطه موج سوم رفتاردرمانى قرار

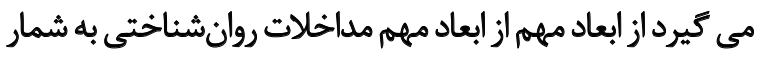

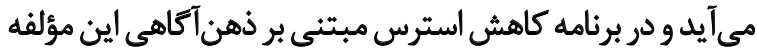

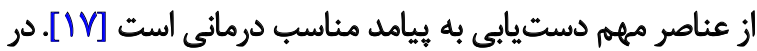

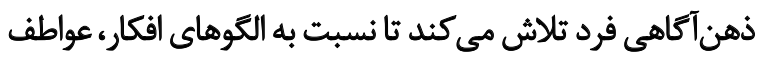

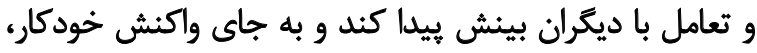

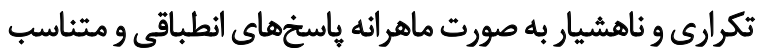

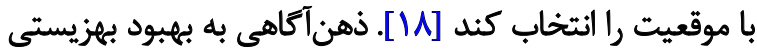

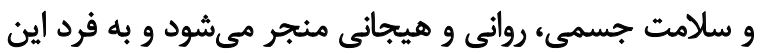

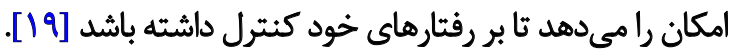
عـوارض ناشـى از بيمـارى مولتييل اسكلروزيس، نار احتى هائي

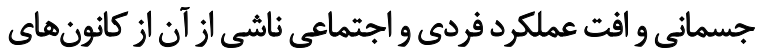

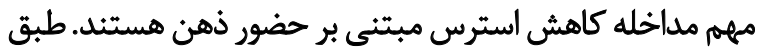

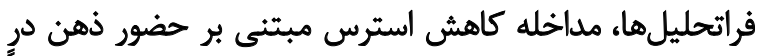

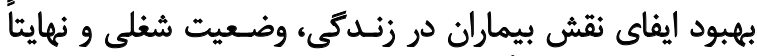

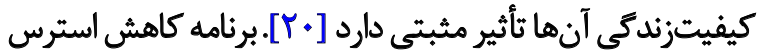

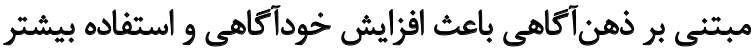

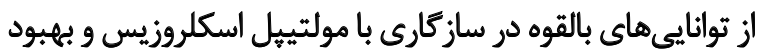

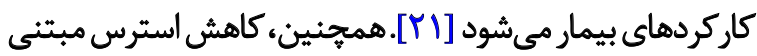

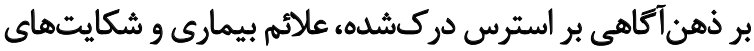

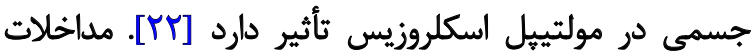

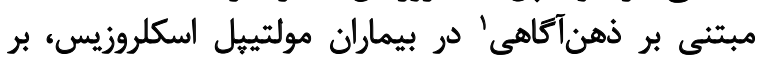

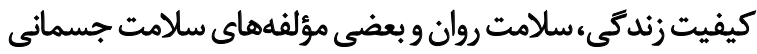

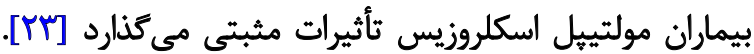

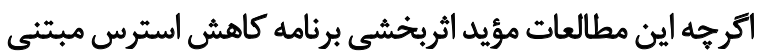

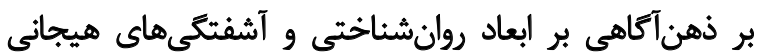

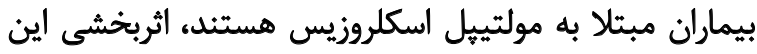

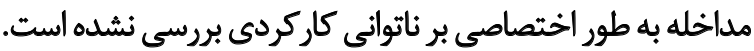

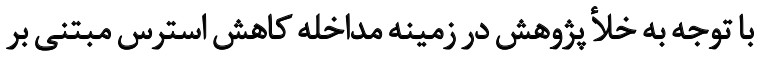

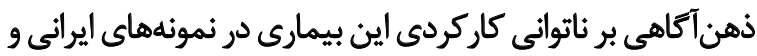

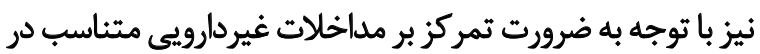

1. Based interventions Mindfulness
آمريكا، در حدود T/ه ميليون نفر در دنيا به اين بيمارى مبتلا

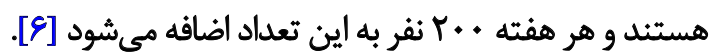
جهار نوع اصلى بيمارى مولتييل اسكلروزيس عبارتاند ازئ

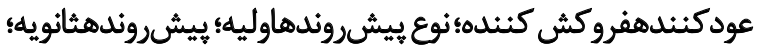

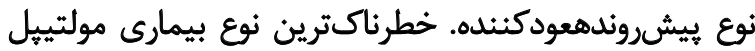

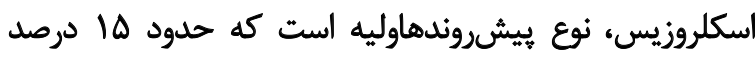

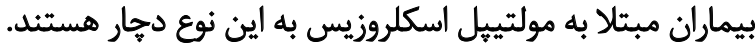

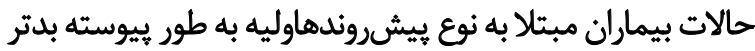

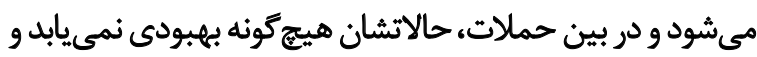

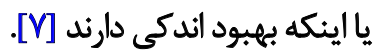

مولتييل اسكلروزيس به عنوان نوعى بيمارى مزمن سبب

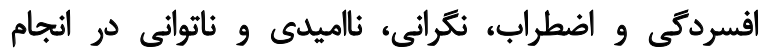

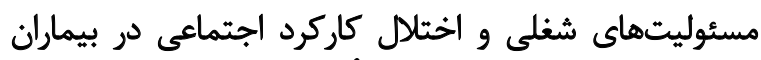

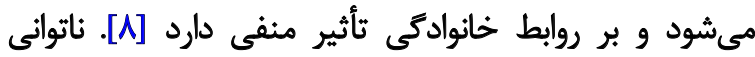

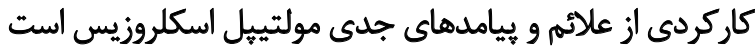

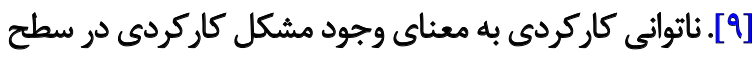

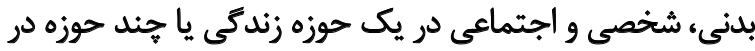

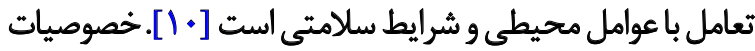

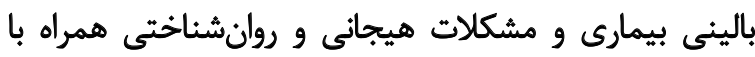

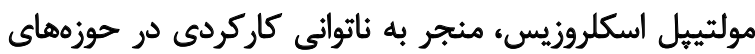

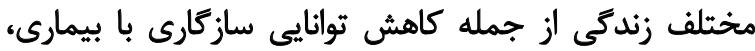

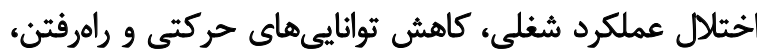

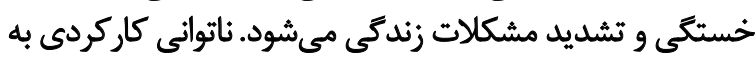

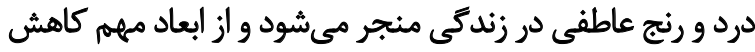

كيفيت زندكى اين بيماران است [ن [11]. مبتلايان به مولتييل اسكلروزيس درجاتي ازئ ناتواني كاركردي

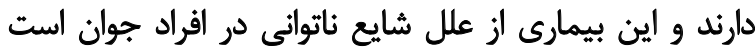

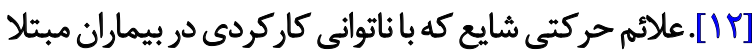

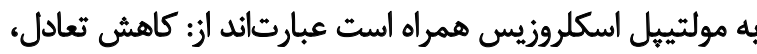

كاهش قدرت، كاهش استقامث و سرعت در راهرفتن [IIT).

به دليل ناتوانى كاركردى، توانايىهاي افراد مبتلا به مولتيتيل

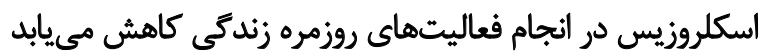

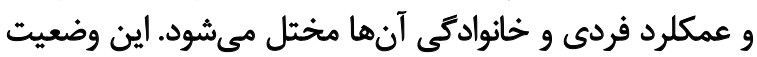

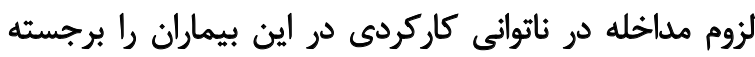

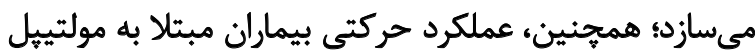

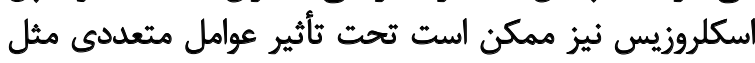

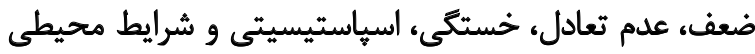

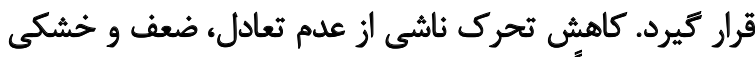

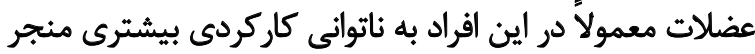

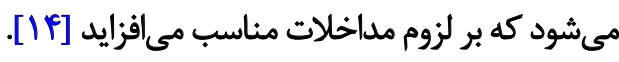

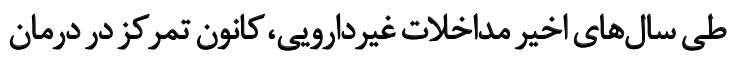
مبتلايان به مولتييل اسكلروزيس بودها است. يكى از اين مداخلات، 
ذهنى، داشتن افكار جدى خودكشى و سابقه سوءمصرف مواد

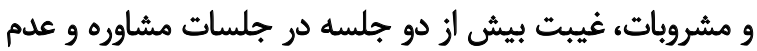
همكارى در فرايند مداخله.

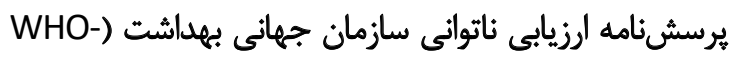

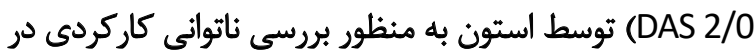

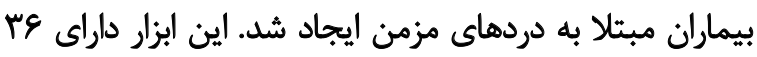

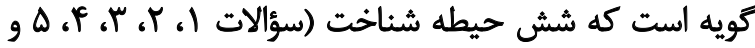

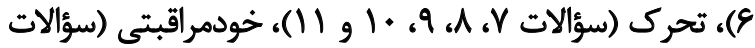

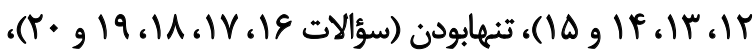

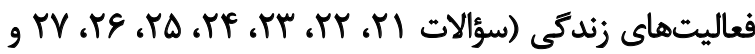

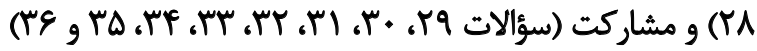

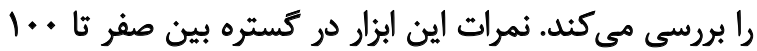

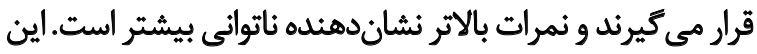

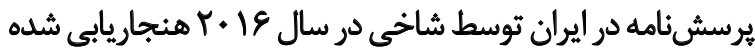

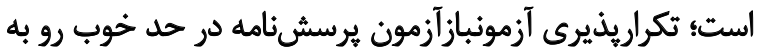

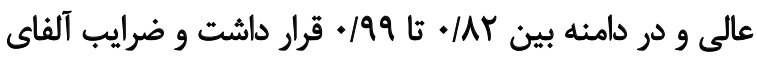

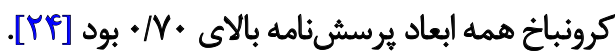
برنامه كاهش استرس مبتنى بر ذهن آكاهى به صورت يك ئري

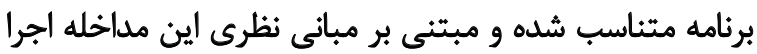

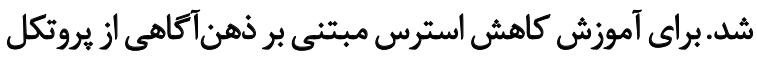

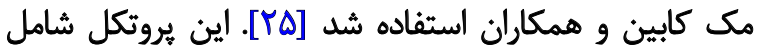

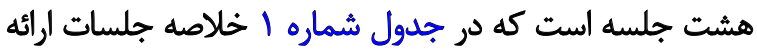

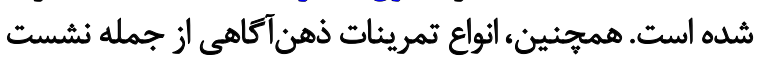

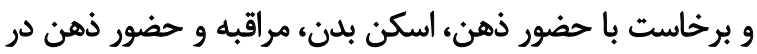

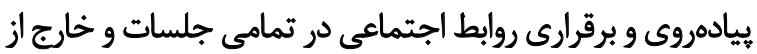

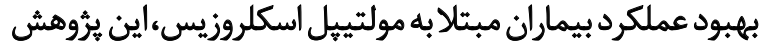

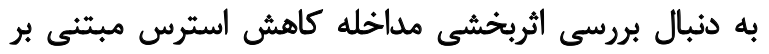

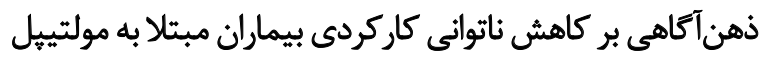
اسكلروزيس است.

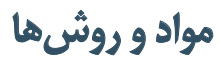

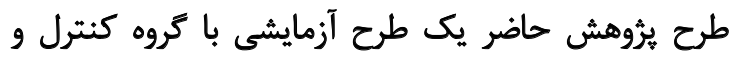

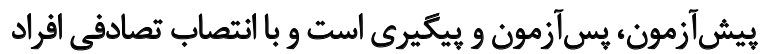

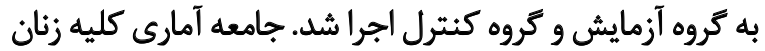

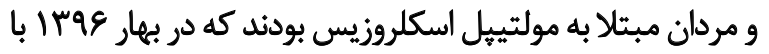

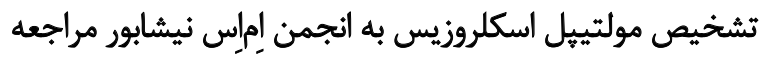

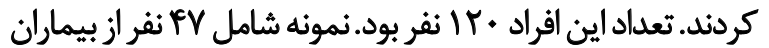

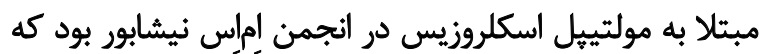

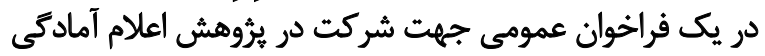

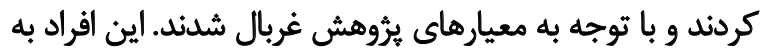

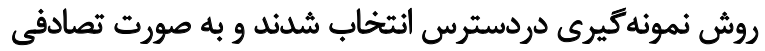

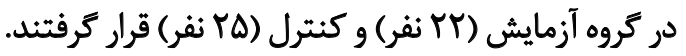

فرمول نمونهيرى بر اساس G-POWER، اندازه اثر و توان

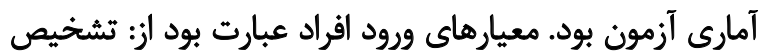

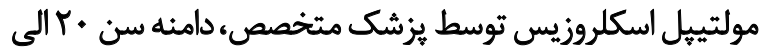

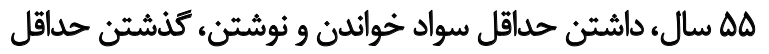

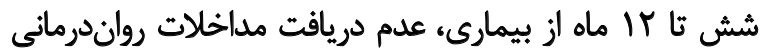

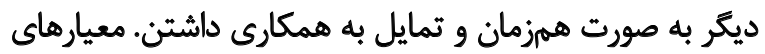

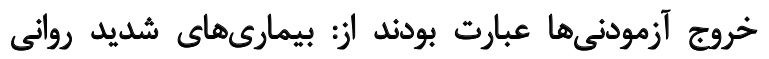

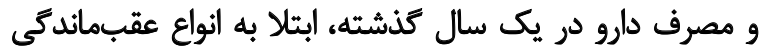

جدول ا. خلاصه جلسات آموزش شناخت درمانى مبتنى بر ذهن آكاهي

\begin{tabular}{|c|c|}
\hline محتواى جلسات أموزشيى & جلسه \\
\hline هدايت خودكار، تمرين تمركز بركشمش همراه با حضور ذهن و مراقبه وارسي بلن & اول \\
\hline مقابله با موانع، تمرين مراقبه وارسى بلن، +1 دقيقه حضور ذهن بر روى جريان تنفس & دوم \\
\hline 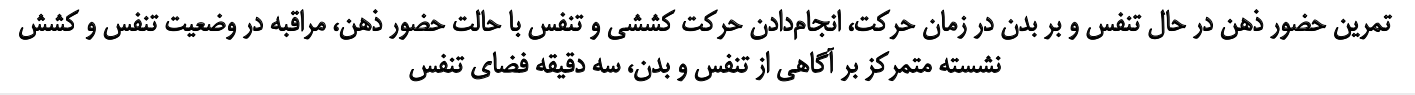 & 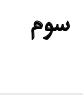 \\
\hline 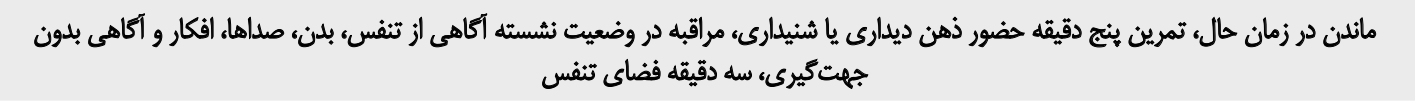 & جهارم \\
\hline 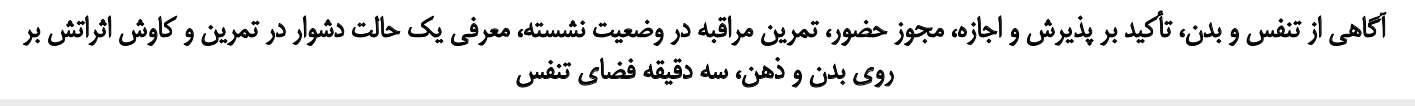 & ينجم \\
\hline 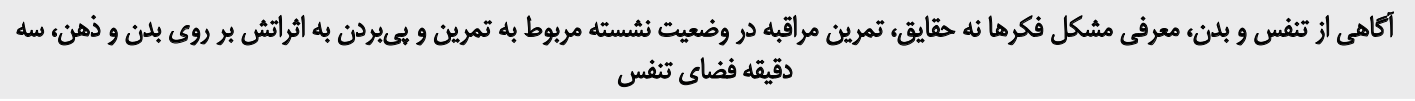 & in \\
\hline 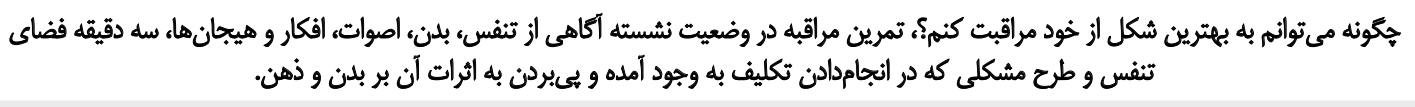 & هفتم \\
\hline استفاده از آموختهها براى كنارآملن با وضعيتهاى خُلقى در آينده، تمرين مراقبه وارسى بلن، جمعبندى، بهايايانرساندن جلسات & هشتم \\
\hline
\end{tabular}


صادق نيست؛ بنابراين براى رعايت اين جيشفرض إز إز اصلاح درجه

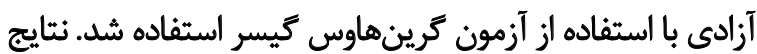

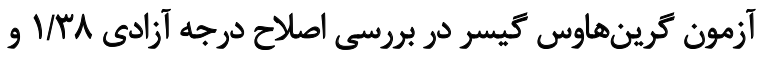

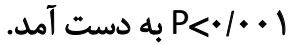

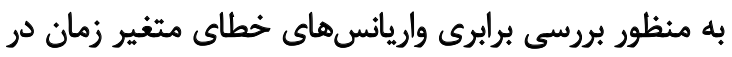

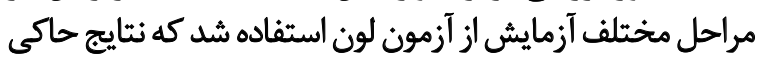

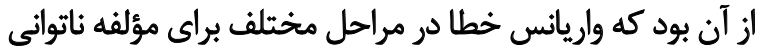

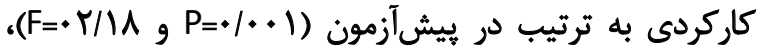

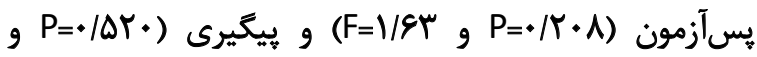
(F=•/Fr.

جدول شماره Y ميانكين و انحراف استاندارد مؤلفههاي مورد

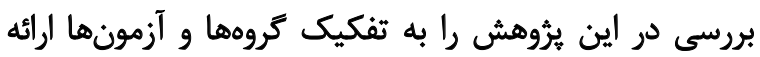

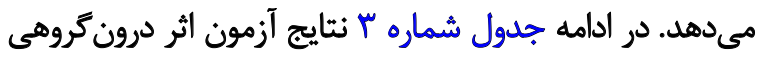

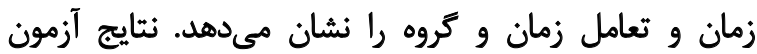

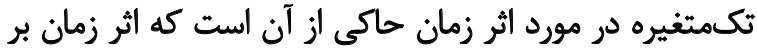

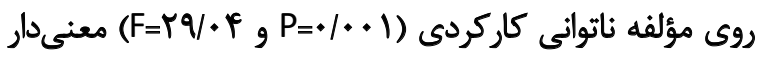

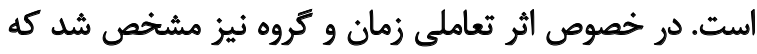

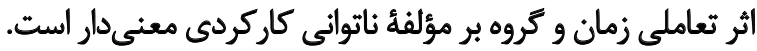
(F=rg/1A, $P=\cdot 1 \cdot \cdot 1)$

نتايج تحليل واريانس بينكروهى نشان مىدهد كه بين دو
جلسات به عنوان تكاليف مداخلهاى انجام شد. دادهها با استفاده

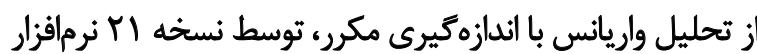

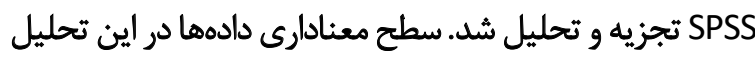

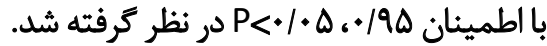

يافتهها

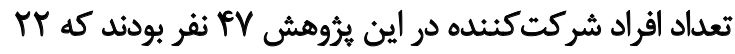

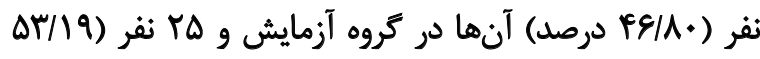

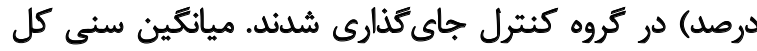

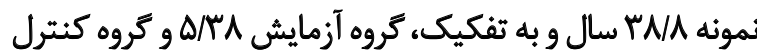

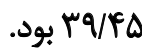

به منظور بررسى اثربخشى كاهش استرس مبتنى بر ذهن آتاهي

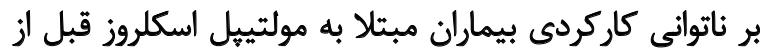

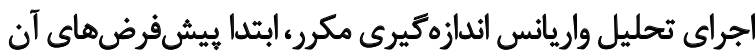

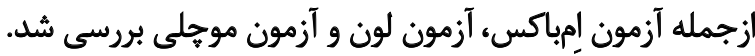

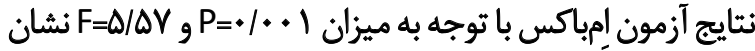

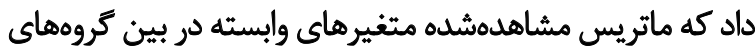

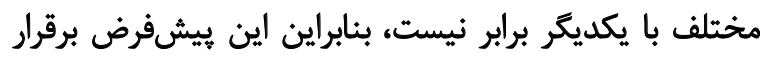

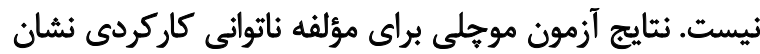

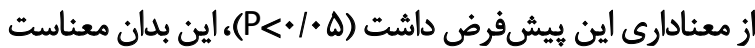

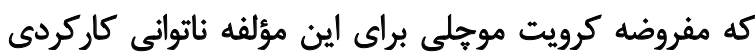

جدول ז. ميانكين و انحراف استاندارد متغيرهاى ئروهش به تفكيك كروه و آزمون

\begin{tabular}{|c|c|c|c|}
\hline ميانكين +انحراف استاندارد & آزمون & ت عروه & مؤلفهـا \\
\hline $\mid r \Delta / r F \pm P / F i$ & ييش أزمون & & \multirow{6}{*}{ ناثوانى كاركردى } \\
\hline $\mid r \Delta / \Delta \Phi \pm 1 \cdot / r+$ & ل يس أزمون & كتنرل & \\
\hline $\mid r r / q \pm q / q 1$ & ييكيرى & & \\
\hline 1.NAE士IA/AF & يشيشآمون & & \\
\hline$\Lambda \Delta / \Lambda) \pm I Y / A Y$ & يس أزمون & آزمايش & \\
\hline$\Lambda \Delta / \Lambda I \pm 1 \cdot / M T$ & يبكيرى & & \\
\hline
\end{tabular}

جدول r. نتايج آزمون اثر نرون كروهى زمان و تعامل زمان و كروه

\begin{tabular}{|c|c|c|c|c|c|c|c|c|c|}
\hline آزمون & مجذور اتا & سطح معنى دارى & $\mathbf{F}$ & ميانكين مجذور & Df & مجموع مجذور & مؤلفه & آزمون & اثر \\
\hline $1 /$. & . & $.1 . .1$ & $r q / . f$ & megpe & I/rA & RTVE/AI & كاركزدئى & كرينهاوس & زمان \\
\hline $1 /$. & (re & $.1+.1$ & re/MA & rAAF/MP & גוא & $r q q \Delta / r T$ & كاركردى & كرينهاوس & ؤرمان \\
\hline
\end{tabular}


جدول F.نتايج تحليل واريانس بين كروهى در ميان دو كروه آزمايش و كنترل

\begin{tabular}{|c|c|c|c|c|c|c|c|c|}
\hline توان أزمون & مجذور اتا & سطح معنى دارى & $\mathbf{F}$ & مجذور ميانكين & Df & مجموع مجذور & مقيلس & منيع \\
\hline \multirow[t]{2}{*}{$1 / .}$. & $\cdot / M A$ & $+1+\infty 1$ & $|S T / 9|$ & $m p r e a / A q$ & 1 & $M r n=\Delta / A q$ & ناثوانى كاركردى & كروه \\
\hline & & & & rIT/gV & Fa & Q & & خطا \\
\hline
\end{tabular}

جدول ه. مقايسه زوجى اثر تعاملى زمان و كروه

\begin{tabular}{|c|c|c|c|c|c|}
\hline سطح معنى دارى & خطاى استاندارد & تفاوت ميانكينها & توره & آزمون & مؤلفه \\
\hline$+/+1$ & $r / .$. & $11 / 4 r$ & بس آزمون & \multirow{2}{*}{ ييش آزمون } & \\
\hline$r /$. & $11 / F r$ & يسأزمون & ييشآزمون & & ناثوانى كاركردى \\
\hline$V / *$ & $1 / . F$ & $+/ \Lambda$ & ييكيرى & يس آزمون & \\
\hline
\end{tabular}

ث

هدف اصلى يروهش حاضر بررسى اثربخشى مداخله كاهش

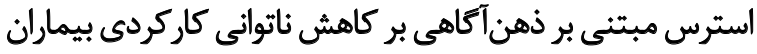

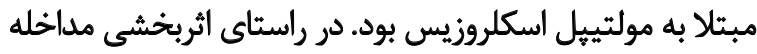

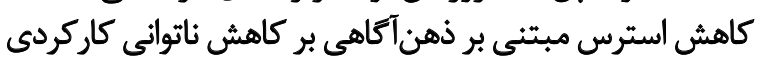

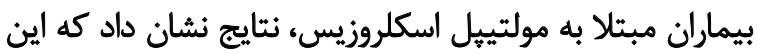

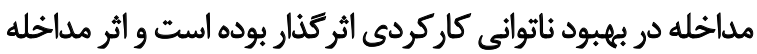

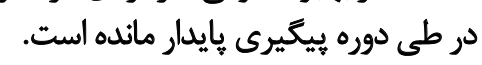

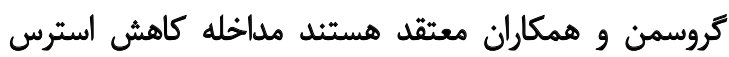

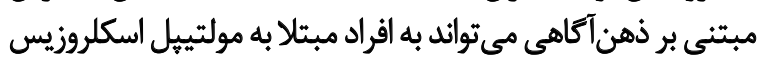

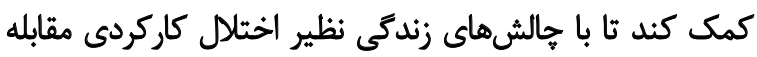

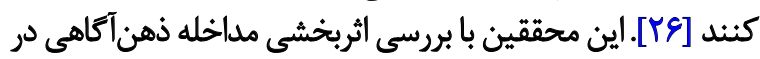

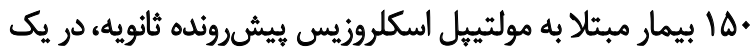

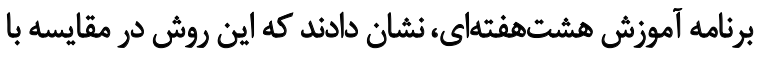

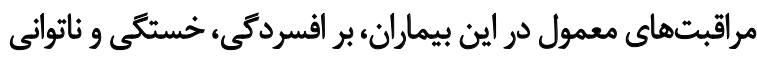

كروه آزمايش و كنترل در مؤلفه ناتوانى كاركردى تفاوت معنادارى

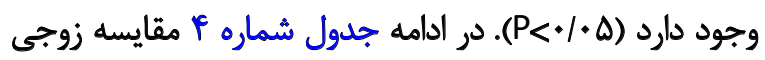

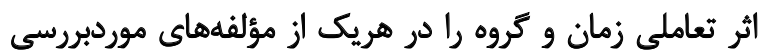

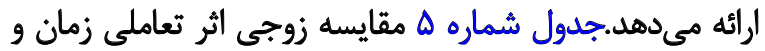

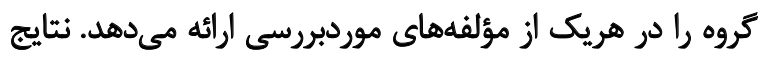

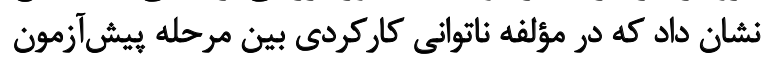

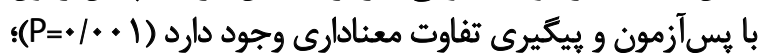

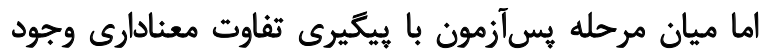

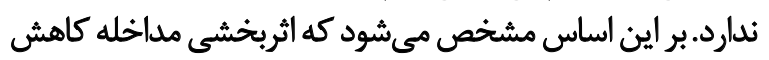

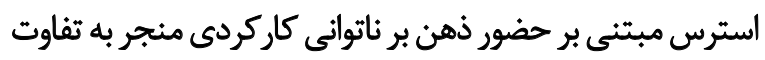

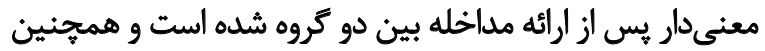

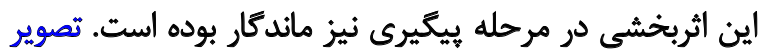

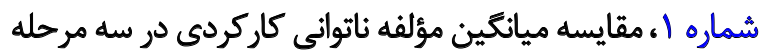

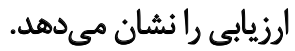

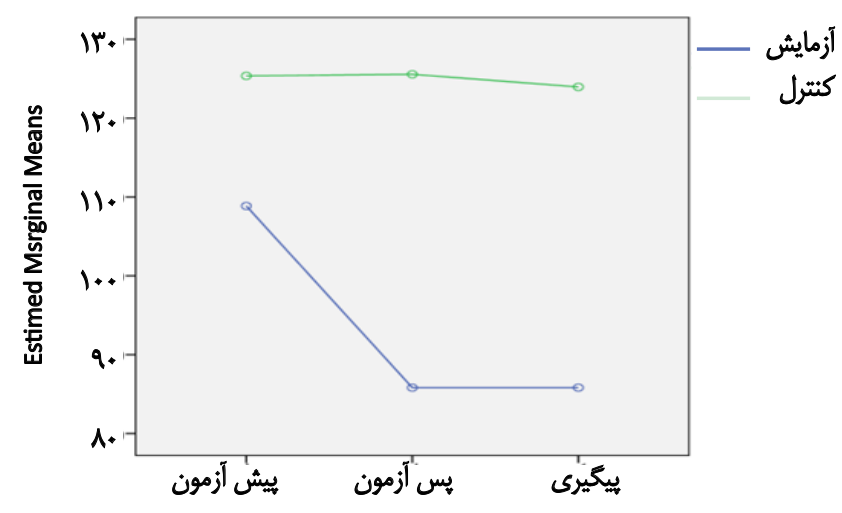

تصوير ا. مقايسه ميانكين مؤلفه ناتوانى كاركرنى در سه مرحله ارزيابى 
مداخله كاهش استرس مبتنى بر ذهن آكاهي ازيكسو باهي باجراي

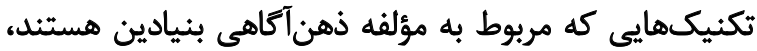

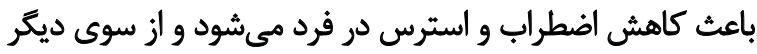

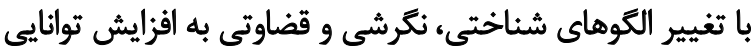

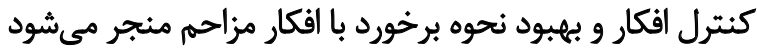

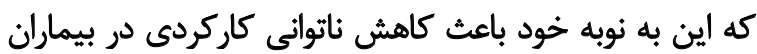

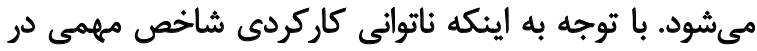

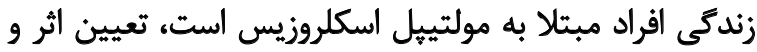

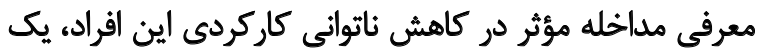
كوشش علمى و اخلاقى تحسين برانغيز است كاني

\section{نتيجليَيرى}

طبق نتايج درمان كاهش استرس مبتنى بر ذهن آكاهي بر بارئي

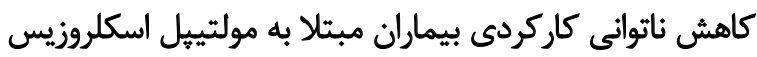

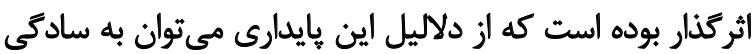

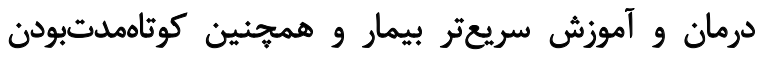

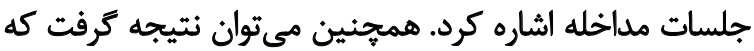

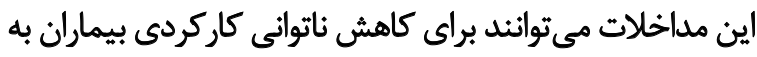
عنوان روشى مفيد مورد استفاده قرار كيرند.

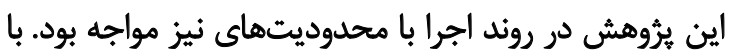

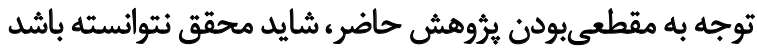

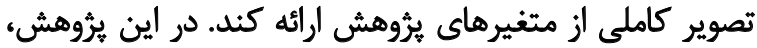

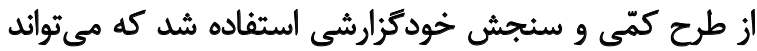

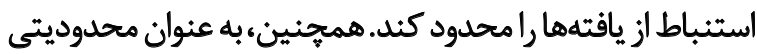
ديكر به علت عدم دسترسى مناسب به به جامعاريه آمارى، امكان امكان

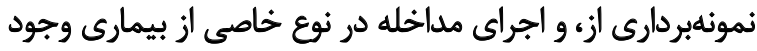

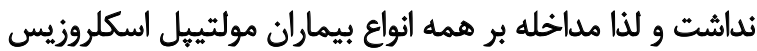

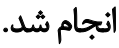

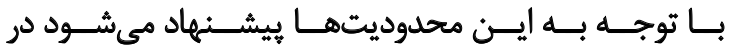

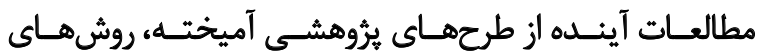

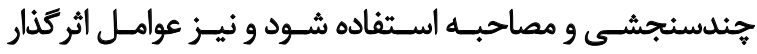

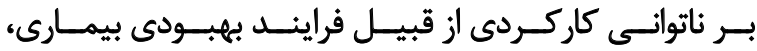

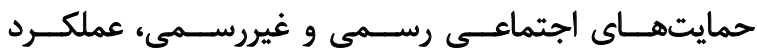

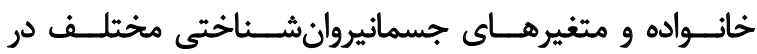

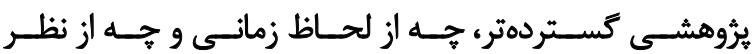

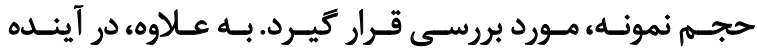

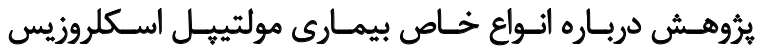

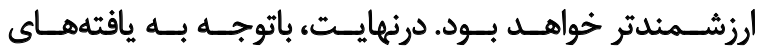

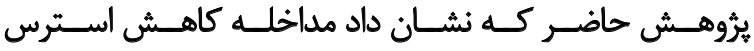

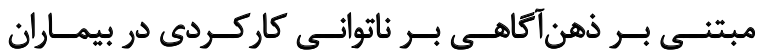

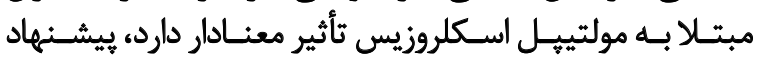

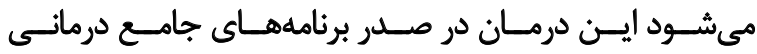

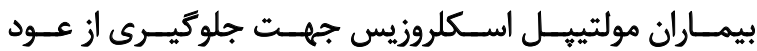

بدنى و كيفيت زندگى آنها تأثير دارد و اين ثاثثيرات در بيكيرى

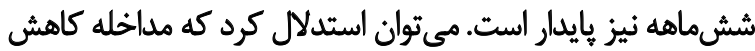

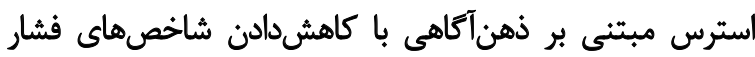

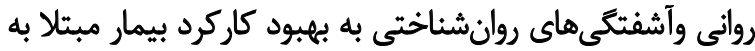

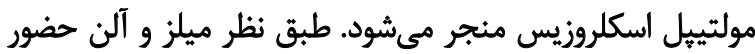

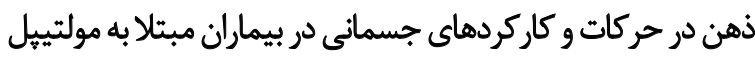

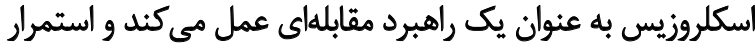

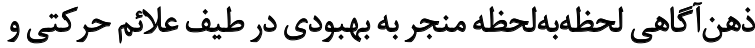

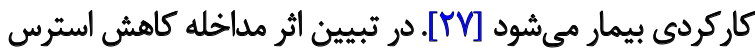

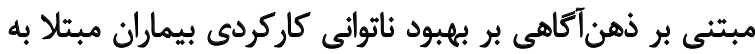

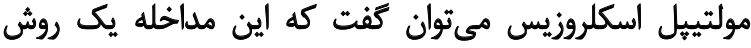

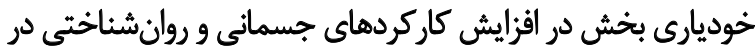

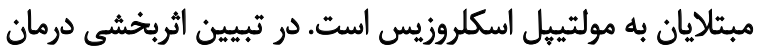

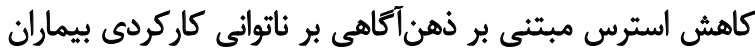

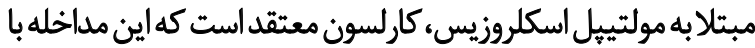

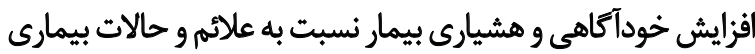

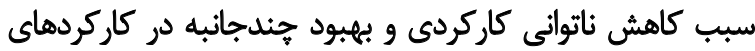

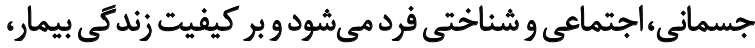

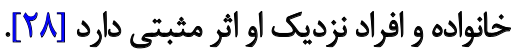
به علاوه، در تبيين ديكرى از نتايج فوق بايد كفت اجراى

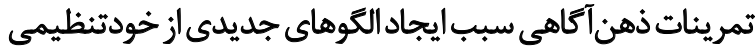

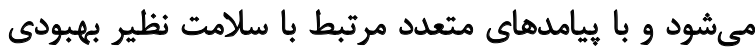

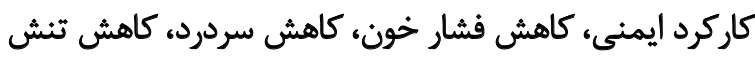

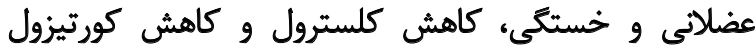

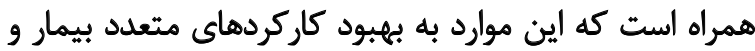

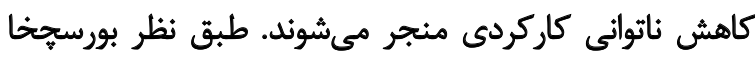

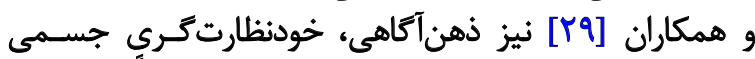

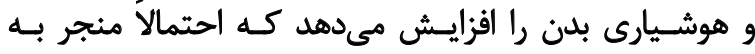

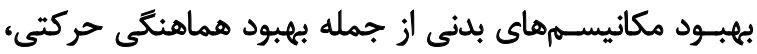

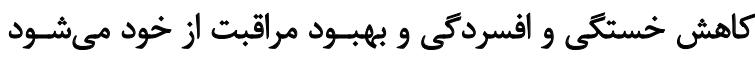

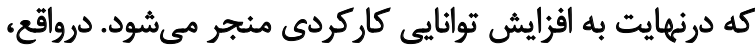

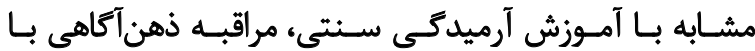

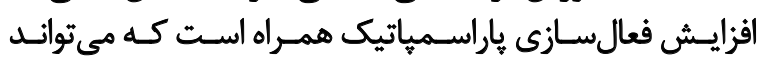

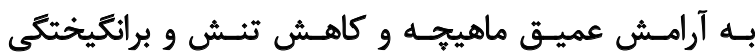
منجـر شـود و زمينه كاركرد مؤثر را فراهم كند.

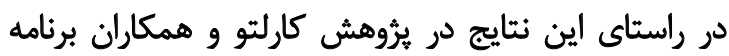

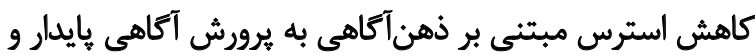

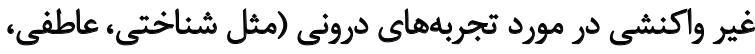

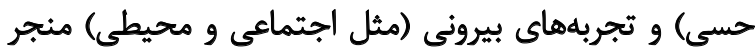

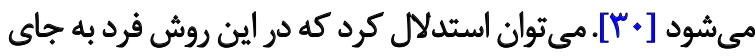

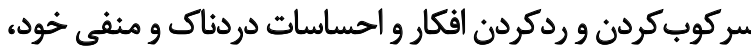

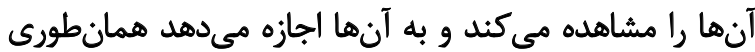

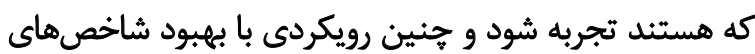
عملكردى همراه است. 


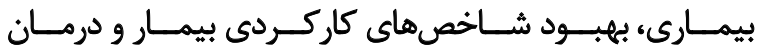

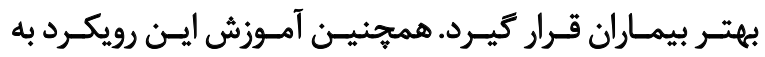

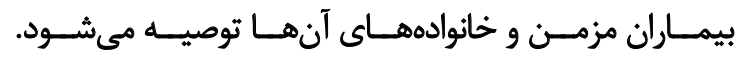
مالاحظات اخلاقى

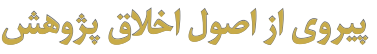

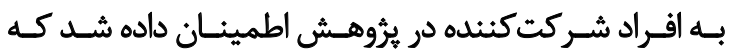

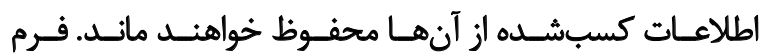

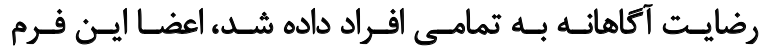

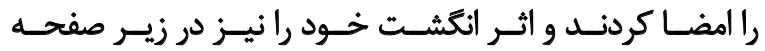

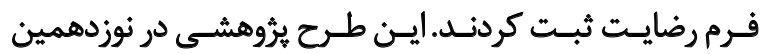

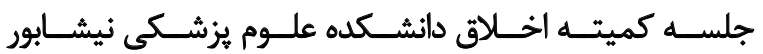

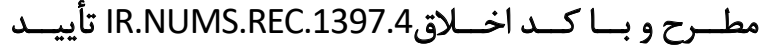

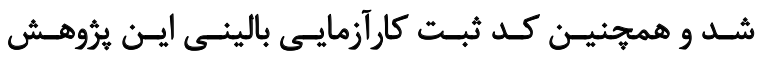
IRCT20151228025732N33

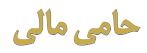

اين مقاله بركرفته از باياينامه كارشناسىارشد نويسنده اول با

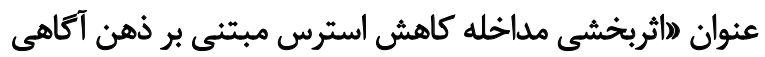

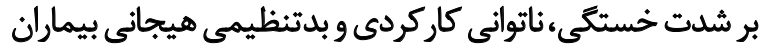

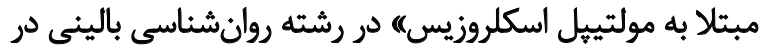

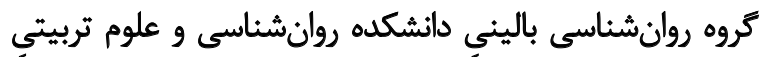

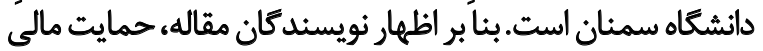

الز ئوهش وجود نداشته است.

\section{مشار كت ثويسنداكان}

تمامى نويسندكان معيارهاى اسثاندارد نويسندكى بر براس اساس

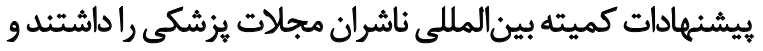
همكى به يك اندازه در نتارش مقاله سهيمه بودند.

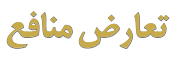

بنابر اظهار نويسندكان اين مقاله تعارض منافع ندارد.

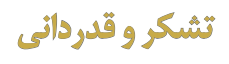

بدينوسيله از حمايتهاي دانشكاه و تمامى دستاندر كارانى كه

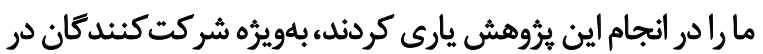

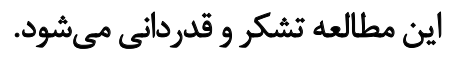




\section{References}

[1] Huang WJ, Chen WW, Zhang X. Multiple sclerosis: Pathology, diagnosis and treatments. Exp Ther Med. 2017; 13(6):3163-6. [DOI:10.3892/ etm.2017.4410] [PMID] [PMCID]

[2] Loma I, Heyman R. Multiple sclerosis: Pathogenesis and treatment. Curr Neuropharmacol. 2011; 9(3):409-16. [DOI:10.2174/157015911796557 911] [PMID] [PMCID]

[3] Ghasemi N, Razavi S, Nikzad E. Multiple sclerosis: Pathogenesis, symptoms, diagnoses and cell-based therapy. Cell J. 2017; 19(1):1-10.

[4] Dobson R, Giovannoni G. Multiple sclerosis, A review. Eur J Neurol. 2019; 26(1):27-40. [DOI:10.1111/ene.13819] [PMID]

[5] Asadpour E, Veisi S. [The Effectiveness of emotionally focused couple therapy on sexual self-esteem and sexual function in women with multiple sclerosis (Persian)]. J Arak Uni Med Sci. 2017; 20 (7):1-12.

[6] Bhise V, Dhib-Jalbut S. Further understanding of the immunopathology of multiple sclerosis: Impact on future treatments. Expert Rev Clin Immunol. 2016; 12(10): 1069-89. [DOI:10.1080/1744666X.2016.1191351] [PMID]

[7] Crayton H, Heyman RA, Rossman HS. A multimodal approach to managing the symptoms of multiple sclerosis. Neurology. 2004; 63(11 Suppl. 5):S12-8. [DOI:10.1212/WNL.63.11_suppl_5.S12] [PMID]

[8] Krokavcova M, Nagyova I, van Dijk JP, Rosenberger J, Gavelova M, Middel $B$, et al. Mastery, functional disability and perceived health status in patients with multiple sclerosis. Eur J Neurol. 2008; 15(11):1237-44. [DOI:10.1111/j.1468-1331.2008.02304.x] [PMID]

[9] Zeqiraj K, Kruja J, Kabashi S, Muçaj S. Epidemiological characteristics and functional disability of multiple sclerosis patients in kosovo. Med Arch. 2014; 68(3):178-81. [DOI:10.5455/medarh.2014.68.178-181] [PMID] [PMCID]

[10] Demir S. Multiple sclerosis functional composite. Noro Psikiyatr Ars. 2018; 55(Suppl. 1):S66-8. [DOI:10.29399/npa.23349]

[11] Soleymani E, Habibi M, Tajoddini E. [The Assessment of cognitive emotion regulation strategies, sensory processing sensitivity and anxiety sensitivity in patients with multiple sclerosis (Persian)]. J Arak Uni Med Sci. 2016; 19 (8):57-66

[12] Kister I, Chamot E, Salter AR, Cutter GR, Bacon TE, Herbert J. Disability in multiple sclerosis: A reference for patients and clinicians. Neurology. 2013; 80(11):1018-24. [DOI:10.1212/WNL.0b013e3182872855] [PMID] [PMCID]

[13] Gilbertson RM, Klatt MD. Mindfulness in motion for people with multiple sclerosis: A feasibility study. Int J MS Care. 2017; 19(5):225-31. [DOI:10.7224/1537-2073.2015-095] [PMID] [PMCID]

[14] Kargarfard M, Shariat A, Ingle L, Cleland JA, Kargarfard M. Randomized controlled trial to examine the impact of aquatic exercise training on functional capacity, balance, and perceptions of fatigue in female patients with multiple sclerosis. Arch Phys Med Rehabil. 2018; 99(2):23441. [DOI:10.1016/j.apmr.2017.06.015] [PMID]

[15] Noonan S. Mindfulness-based stress reduction. Can Vet J. 2014; 55(2):134-5.

[16] Blankespoor RJ, Schellekens MPJ, Vos SH, Speckens AEM, de Jong BA. The effectiveness of mindfulness-based stress reduction on psychological distress and cognitive functioning in patients with multiple sclerosis: A pilot study. Mindfulness (NY). 2017; 8(5):1251-8. [DOI:10.1007/ s12671-017-0701-6] [PMID] [PMCID]
[17] Willekens B, Perrotta G, Cras P, Cools N. Into the moment: Does mindfulness affect biological pathways in multiple sclerosis? Front Behav Neurosci. 2018; 12:103. [DOI:10.3389/fnbeh.2018.00103] [PMID] [PMCID]

[18] Kolahkaj B, Zargar F. Effect of mindfulness-based stress reduction on anxiety, depression and stress in women with multiple sclerosis. Nurs Midwifery Stud. 2015; 4(4):1-7. [DOI:10.17795/nmsjournal29655] [PMID] [PMCID]

[19] Nejati S, Rajezi Esfahani S, Rahmani S, Afrookhteh G, Hoveida S. The effect of group mindfulness-based stress reduction and consciousness yoga program on quality of life and fatigue severity in patients with ms. J Caring Sci. 2016; 5(4):325-35. [DOI:10.15171/jcs.2016.034] [PMID] [PMCID]

[20] Simpson R, Booth J, Lawrence M, Byrne S, Mair F, Mercer S. Mindfulness based interventions in multiple sclerosis, a systematic review. BMC Neurol. 2014; 14:15. [DOI:10.1186/1471-2377-14-15] [PMID] [PMCID]

[21] Fayazi B, Parnow A, Ahsan B, Yousefvand Z. [The Effect of aerobic training on functional capacity and percent body fat in women with moderate multiple sclerosis (Persian)]. J Arak Uni Med Sci. 2013; 16(1):87-97.

[22] Crescentini C, Matiz A, Cimenti M, Pascoli E, Eleopra R, Fabbro F. Effect of mindfulness meditation on personality and psychological well-being in patients with multiple sclerosis. Int J MS Care. 2018; 20(3):101-8. [DOI:10.7224/1537-2073.2016-093] [PMID] [PMCID]

[23] Malinowski P. Neural mechanisms of attentional control in mind fulness meditation. Fornt Neurosci. 2013; 7:8-18. [DOI:10.3389/ fnins.2013.00008] [PMID] [PMCID]

[24] Salehi R, Shakhi K, Faraji Khiavi F. Association between disability and quality of life in multiple sclerosis patients in Ahvaz, IRAN. Master Sociomed. 2016; 28(3):215-9. [DOI:10.5455/msm.2016.28.215-219] [PMID] [PMCID]

[25] Mccubbin T, Dimidjian S, Kempe K, Glassey MS, Ross C, Beck A Mindfulness-based stress reduction in an integrated care delivery system: One-year impacts on patient-centered outcomes and health care utilization. Perm J. 2014; 18(4):4-9. [DOI:10.7812/TPP/14-014] [PMID] [PMCID]

[26] Grossman P, Niemann L, Schmidt S, Walach H. Mindfulness-based stress reduction and health benefits, a meta-analysis. J Psychosom Res. 2004; 57(1):35-43. [DOI:10.1016/S0022-3999(03)00573-7]

[27] Mills N, Allen J. Mindfulness of movement as a coping strategy in multiple sclerosis. A pilot study. Gen Hosp Psychiatry. 2000; 22(6): 425-31. [DOI:10.1016/S0163-8343(00)00100-6]

[28] Carlson LE. Mindfulness-based interventions for physical conditions: A narrative review evaluating levels of evidence. ISRN Psychiatry. 2012; 2012:1-14. [DOI:10.5402/2012/651583] [PMID] [PMCID]

[29] Burschka JM, Keune PM, Oy UH, Oschmann P, Kuhn P. Mindfulnessbased interventions in multiple sclerosis: Beneficial effects of Tai Chi on balance, coordination, fatigue and depression. BMC Neurol. 2014; 14:19. [DOI:10.1186/s12883-014-0165-4] [PMID] [PMCID]

[30] Carletto S, Borghi M, Francone D, Scavelli F, Bertino G, Cavallo M et al. The efficacy of a Mindfulness Based Intervention for depressive symptoms in patients with Multiple Sclerosis and their caregivers: Study protocol for a randomized controlled clinical trial. BMC Neurol. 2016 16:1-8. [DOI:10.1186/s12883-016-0528-0] [PMID] [PMCID] 
This Page Intentionally Left Blank 